\title{
Respiratory Acidosis
}

National Cancer Institute

\section{Source}

National Cancer Institute. Respiratory Acidosis. NCI Thesaurus. Code C50728.

A condition in which the blood pH is less than normal, secondary to impaired gas exchange. 